\title{
Modelagem e controle de sistemas públicos de compartilhamento de bicicletas utilizando modelos em redes de Petri ${ }^{\star}$
}

\author{
Braian Igreja de Freitas* João Carlos Basilio* \\ * COPPE - Programa de Engenharia Elétrica, Universidade Federal do \\ Rio de Janeiro, RJ (e-mail: bfreitas@poli.ufrj.br,basilio@dee.ufrj.br)
}

\begin{abstract}
Bicycle sharing has, as principle, to allow people to travel between two points using a bicycle, being public bicycle sharing systems (PBSS) originated from. PBSS are formed with stations where users can pick up bicycles to move themselves around the stations. Depending on the usage of the stations, they may have all the bicycles removed from them or may become full, restricting the movement of bicycles through the stations. One way to solve this issue is to implement balancing strategies by using a vehicle to exchange bicycles between stations, balancing them. In this paper, we proposed a model of a generic PBSS using Petri nets in order to evaluate different strategies based on a single simulation algortithm. The proposed model is then validated by an analyze of the design of Integra UFRJ PBSS system with the combination of three different balancing strategies, also proposed in this paper.

Resumo: Compartilhamento de bicicletas possui como princípio possibilitar que um indivíduo trafegue de um ponto a outro com bicicletas. Com base nessa concepção, originaram-se os sistemas públicos de compartilhamento de bicicletas (SPCB), que consistem de estações onde os usuários podem retirar bicicletas para se locomoverem entre elas. Dependendo do uso das estações, elas podem ficar vazias ou lotadas, restringindo a movimentação de bicicletas nelas. Uma maneira de solucionar esse problema é implementar estratégias de balanceamento utilizando um veículo para realizar trocas de bicicletas entre as estações, equilibrando-as. Neste artigo, será proposto um modelo de um SPCB genérico em redes de Petri para avaliar diferentes estratégias em um único algoritmo de simulação. O modelo proposto será validado pela análise do desempenho do projeto de SPCB Integra UFRJ com a combinação de três tipos diferentes de estratégias de balanceamento formuladas neste artigo.
\end{abstract}

Keywords: Discrete event systems, Petri net, PBSS; transportation; redistribution.

Palavras-chaves: Sistemas a eventos discretos, Redes de Petri, SPCB, transportes, redistribuição.

\section{INTRODUÇÃO}

Os sistemas públicos de compartilhamento de bicicletas (SPCB) têm como princípio permitir que um indivíduo possa retirar uma bicicleta de um lugar e devolvê-la a outro, possibilitando que ele trafegue de um ponto a outro. Cada SPCB é composto por estações, cada uma com um número fixo de vagas de bicicletas.

Durante o funcionamento do SPCB, os usuários retiram bicicletas das estações que possuam pelo menos uma bicicleta disponível e as depositam nas suas estações de destino que devem possuir pelo menos uma vaga de bicicleta livre. Assim, para que um SPCB possa atender às necessidades de mobilidade dos seus usuários em uma dada região com maior êxito, seus parâmetros, tais como quantidade e localização de estações e o número de vagas de bicicletas de cada estação, devem ser bem planejados.

\footnotetext{
* Este trabalho foi financiado em parte pela Coordenação de Aperfeiçoamento de Pessoal de Nível Superior (CAPES), Código de Financiamento 001, e pelo Conselho Nacional de Desenvolvimento Científico e Tecnológico (CNPq), processo número 309652/2017-0.
}

Apesar do bom planejamento do sistema ajudar no seu funcionamento, o fluxo de usuários entre as estações não é uniforme ao longo do dia. Por isso, dois problemas podem surgir: muitos usuários podem desejar sair de uma mesma estação simultaneamente, retirando bicicletas até que ela fique sem bicicletas, ou muitos usuários podem viajar para o mesmo destino, lotando a estação e impedindo que outros ciclistas depositem nela as suas bicicletas.

Para evitar que a quantidade de bicicletas nas estações sejam alterados apenas pelos usuários, alguns sistemas adotam a utilização de veículos, para realizar a redistribuição de bicicletas entre as estações, cuja função é retirar bicicletas das estações que estão cheias de bicicletas e repor bicicletas nos locais que estão vazios por meio de rondas executadas durante o dia entre as estações. Nesse sentido, diversos estudos de algoritmos de balanceamento foram propostos (Chemla et al., 2013; Vogel and Mattfeld, 2010; Shu et al., 2010; Benarbia et al., 2013) e de modelos de SPCB (Vogel et al., 2011; Labadi et al., 2012, 2014; Antunes et al., 2016). 
Neste artigo será proposto um modelo em redes de Petri capaz de não só reproduzir o comportamento do SPCB real como também analisar o desempenho do sistema em função de diferentes estratégias de balanceamento. Para tanto, será proposto as redes de Petri estocásticas com controles externos, que permitem modelar tanto um sistema a eventos discretos por redes de Petri como também algoritmos externos de controle.

Para validar a abordagem proposta, será utilizado o SPCB recentemente implementado na Ilha do Fundão da cidade Rio de Janeiro, o projeto Integra UFRJ.

\section{CONCEITOS TEÓRICOS}

Uma rede de Petri é um grafo bipartido ponderado definido pela quádrupla $\mathcal{N}=(P, T$, Pre, Post) (Murata, 1989; David and Alla, 1994), em que $P=\left\{p_{1}, p_{2}, \ldots, p_{m}\right\}$ é o conjunto finito de lugares, $T=\left\{t_{1}, t_{2}, \ldots, t_{n}\right\}$ é o conjunto finito de transições, Pre (resp. Post) é uma matriz $m \times n$ cujo elemento $\operatorname{Pre}(i, j)$ (resp. $\operatorname{Post}(i, j))$ representa o peso do arco que liga o lugar $p_{i}$ (resp. transição $t_{i}$ ) com a transição $t_{j}$ (resp. lugar $p_{j}$ ). Os conjuntos de entradas de uma transição $t_{j}$ (resp. um lugar $p_{i}$ ) são denominados lugares (resp. transições) de entrada e são denotados por $I\left(t_{j}\right)$ (resp. $\left.I\left(p_{i}\right)\right)$. Analogamente, os lugares e transições de saída são denotados por $O\left(t_{j}\right)$ e $O\left(p_{i}\right)$, respectivamente.

$\mathrm{O}$ estado atual de uma rede de Petri é representado por um vetor de marcações de lugares $\underline{m}=\left[m\left(p_{1}\right) m\left(p_{2}\right) \ldots\right.$ $\left.m\left(p_{n_{P}}\right)\right]^{T}$, cujos componentes $m\left(p_{i}\right)$ representam a quantidades de fichas em cada lugar $p_{i} \in P$. O estado inicial de uma rede de Petri é representado por $\underline{m}_{0}$. Uma transição $t_{j}$ está habilitada quando o número de fichas de cada um dos seus lugares de entrada for maior ou igual do que os pesos dos arcos que ligam esses lugares à transição $t_{j}$. Quando uma transição $t_{j}$, que está habilitada, dispara, as fichas se movimentam de acordo a seguinte equação

$$
m^{\prime}\left(p_{i}\right)=m\left(p_{i}\right)-\operatorname{Pre}\left(p_{i}, t_{j}\right)-\text { Post, } \forall p_{i} \in P\left(t_{j}, p_{i}\right),
$$

em que $m\left(p_{i}\right)$ e $m^{\prime}\left(p_{i}\right)$ denotam as marações do lugar $p_{i}$ antes e depois do disparo de $t_{j}$, respectivamente.

Para que o disparo da transição seja determinado por um intervalo de tempo que deve transcorrer desde o instante em que a transição foi habilitada, o conjunto de transições $T$ deve ser particionado nos conjuntos $T_{i}$ e $T_{d}$, que são formados, respectivamente, pelas transições imediatas, que disparam assim que habilitadas, e pelas transições temporizadas, que disparam após a passagem de um tempo depois da sua habilitação. Graficamente, as transições temporizadas são caixas retangulares vazias.

Redes de Petri podem possuir lugares com mais de uma transição de saída. Essa ocorrência é chamada de conflito estrutural (David and Alla, 1994), sendo denotado pela dupla $C\left(p,\left\{t_{1}, t_{2}, \ldots, t_{k}\right\}\right)$ formada pelo lugar $p$ e pelo conjunto $\left\{t_{1}, t_{2}, \ldots, t_{k}\right\}$, das transições que formam o conflito.

Com o objetivo de adicionar características probabilísticas aos disparos das transições, torna-se necessário utilizar as chamadas redes de Petri estocásticas generalizadas (RPEG), que permitem: (i) atribuir a transições temporizadas, tempos de disparos definidos estocasticamente; (ii) resolver conflitos entre transições imediatas por meio de probabilidades. As RPEG são definidas pela sétupla
Tabela 1. Interpretações dos parâmetros da rede de Petri proposta.

\begin{tabular}{ll}
\hline Parâmetro & Interpretação \\
\hline$R_{i}$ & Número de reordenação de cada estação $S_{i} \cdot$ \\
$C_{i}$ & Capacidade da estação $S_{i}$. \\
$C_{v}$ & Capacidade do veículo de redistribuição. \\
$N_{B}$ & Número total de bicicletas no SPCB. \\
\hline
\end{tabular}

$\mathcal{N E}=\left(\mathcal{N}, \underline{m}_{0}, \Lambda_{d}, \Lambda_{i}\right)$, sendo $\Lambda_{d}$ e $\Lambda_{i}$ definidos como: para cada transição $t_{j} \in T_{d}$ é definida um função densidade de probabilidade que determina estocasticamente o intervalo de disparo, e para cada transição $t_{j} \operatorname{em} C\left(p,\left\{t_{1}, t_{2}, \ldots, t_{k}\right\}\right)$ é atribuída uma probabilidade $\Lambda_{i}\left(t_{j}, p\right)$ de disparo de $t_{j}$ dado que as transições $t_{1}, t_{2}, \ldots, t_{k}$ estão habilidades por $p$. Formalmente, $\lambda_{d}$ é definido, para $t \in T_{d}$, como

$$
\Lambda_{d}(t)=f d p(x)
$$

em que $x$ é uma variável aleatória tal que a probabilidade $p\left(x \leq d_{t}\right)=\int_{0}^{d_{t}} f d p_{t_{j}}(x) d x$, sendo $d_{t}$ o tempo de retardo de disparo da transição $t_{j}$. Em contrapartida, $\lambda_{i}$ é formalmente definido como

$$
\Lambda_{i}(t, p)=P R C(t \mid p), \forall t \in C\left(p,\left\{t_{1}, t_{2}, \ldots, t_{k}\right\}\right),
$$

em que $P R C\left(t_{j} \mid p\right)$ representa, para cada transição $t_{j} \in T_{i}$ envolvida no conflito em $p$, a probabilidade dessa transição ser escolhida para disparar. Note que a soma de todos os $\operatorname{PRC}\left(t_{j} \mid p\right)$ do conflito $C\left(p,\left\{t_{1}, t_{2}, \ldots, t_{k}\right\}\right)$ é igual a 1 .

Caso um conflito estrutural $C\left(p,\left\{t_{1}, t_{2}, \ldots, t_{k}\right\}\right)$ precise ser resolvido por meio de um agente externo, torna-se necessário estender a RPEG de forma a levar em conta a atuação do agente externo. A essa nova rede de Petri dá-se o nome de rede de Petri estocástica generalizada com controle externo (RPEGCE), que é definida como $\mathcal{N C}=(\mathcal{N E}, E s c)$, sendo $E s c\left(t_{c}, p\right)=1$ para a transição $t_{c} \in\left\{t_{1}, t_{2}, \ldots, t_{k}\right\}$ escolhida para disparo e $E s c(t, p)=0$, para as demais.

\section{MODELO PROPOSTO PARA UM SPCB}

O modelo em RPEGCE proposto neste artigo estende aquele proposto em Antunes et al. (2016), e permite simular e avaliar o desempenho de SPCB com $N$ estações, $S_{1}, S_{2}, \ldots, S_{N}$, e um veículo de redistribuição, o qual é capaz de remanejar um certo número de bicicletas nas visitas realizadas às estações em diferentes momentos do dia. Um agente externo definirá a ordem em que as visitas às estações serão realizadas, podendo, inclusive variar de acordo com o estado atual do sistema. A figura 1 mostra a rede de Petri do modelo da estação $S_{1}$ de um SPCB com três estações. Os correspondentes lugares, transições e parâmetros são definidos, respectivamente, pelas tabelas $1,2,3$. Note que podem ser destacadas três sub-redes:

- Sub-rede do fluxo de bicicletas (Lugares pretos). Representa a movimentação das bicicletas entre as estações pelos usuários. Como em um SPCB, cada estação $S_{i}$ possui um número fixo de vagas $C_{i}$ que são ocupadas por bicicletas ao longo do funcionamento do sistema, torna-se necessário definir os lugares $P B S_{i}$ e $P E S_{i}$ para representarem, respectivamente, o número de bicicletas que ocupam a estação e o número de vagas disponíveis na estação. Se um usuário desejar alugar uma bicicleta da estação $S_{i}$ e a estação possuir pelo menos uma bicicleta disponível, ou seja, $m\left(P B S_{i}\right) \geq 1$, ele irá retirar a bicicleta da estação. Essa ação é representada pelo disparo da 


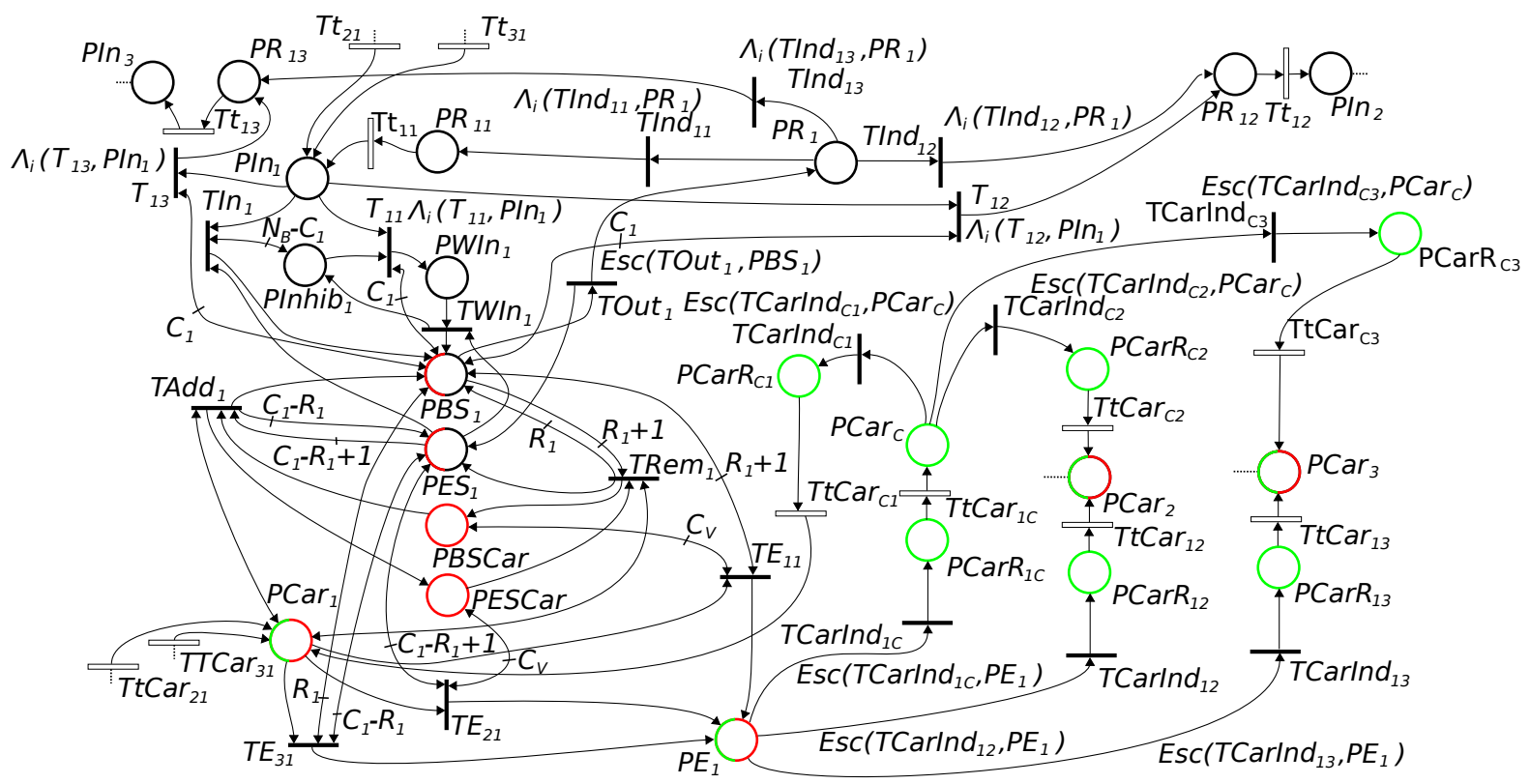

Figura 1. Rede de Petri estocástica generalizada com controle externo da estação $S_{1}$ de um SPCB com três estações.

Tabela 2. Interpretações dos lugares da rede de Petri proposta.

\begin{tabular}{|c|c|}
\hline Lugar & Interpretação \\
\hline$P I n_{i}$ & $\begin{array}{l}\text { Representa as bicicletas dos usuários que desejam estaci- } \\
\text { onar na estação } S_{i} \text {. }\end{array}$ \\
\hline PInhib $_{i}$ & $\begin{array}{l}\text { Impede, através da perda de fichas, que outros usuários } \\
\text { depositem bicicletas a estação } S_{i} \text { ao chegar nelas. }\end{array}$ \\
\hline$P E_{i}$ & $\begin{array}{l}\text { Indica o término do balanceamento na estação } S_{i} \text { pelo } \\
\text { veículo de redistribuição. }\end{array}$ \\
\hline$P E S_{i}$ & os suportes vazios da estação $S_{i}$. \\
\hline$P B S_{i}$ & le de bicicletas na estação $S_{i}$. \\
\hline$P R_{i}$ & Representa as bicicletas que saíram da estação $S_{i}$. \\
\hline$P W I n_{i}$ & $\begin{array}{l}\text { Indica quantos usuários estão aguardando por vagas de } \\
\text { bicicletas em } S_{i} \text {, quando } S_{i} \text { estiver lotada. }\end{array}$ \\
\hline $\mathrm{PCar}_{i}$ & O veículo de redistribuição está \\
\hline$P R_{i j}$ & $\begin{array}{l}\text { s bicicletas que saíram da estação } S_{i} \text { com } \\
\text { ação } S_{j} \text {. }\end{array}$ \\
\hline PCar & $\begin{array}{l}\text { iniu que o veículo de redistribuição deve se } \\
\text { para o centro de redistribuição. }\end{array}$ \\
\hline PCar & $\begin{array}{l}\text { ículo de redistribuição deve se } \\
S_{j} .\end{array}$ \\
\hline $\mathrm{PCar}_{C}$ & $\begin{array}{l}\text { Informa se o veículo de redistribuição está no centro de } \\
\text { redistribuição. }\end{array}$ \\
\hline $\operatorname{PCarR}_{C j}$ & $\begin{array}{l}\text { O sistema definiu que o veículo de redistribuição deve se } \\
\text { mover do centro de redistribuição para } S_{j} \text {. }\end{array}$ \\
\hline PES & Rep \\
\hline$P B S$ & Representa a quantidade de bicicletas no veículo. \\
\hline
\end{tabular}

transição TOut $_{i}$ que adiciona uma ficha ao lugar $P R_{i}$. A escolha para qual estação o usuário irá será determinada probabilisticamente a partir dos valores $\Lambda_{i}\left(T I n d_{i j}, P R_{i}\right)$ para fazer a escolha da estação $S_{j}$ como destino, decisão essa que é efetivada pelo disparo da transição $T I n d_{i j}$, que move a ficha para o lugar $P R_{i j}$.

Decorrido um intervalo tempo $\Lambda_{d}\left(T t_{i j}\right)$, que representa o tempo que o usuário leva para se deslocar da estação $S_{i}$ para $S_{j}$, a transição $T t_{i j}$ dispara, deslocando a ficha que representa esse usuário para o lugar $P I n_{j}$. No lugar $P I n_{j}$, é verificado se existe alguma ficha em $P E S_{j}$ e se o lugar $P I n h i b_{j}$ não perdeu uma ficha, verificando se não há nenhum outro usuário esperando por vagas na estação. Caso ambas as verificações sejam válidas, $T I n_{j}$ dispara, representando que a bicicleta foi depositada na estação. Caso a transição $T I_{n}$ não esteja habilitada
Tabela 3. Interpretações das transições da rede de Petri proposta.

\begin{tabular}{|c|c|}
\hline Transição & Interpretação \\
\hline TOut $_{i}$ & Um usuário retira uma bicicleta da estação $S_{i}$. \\
\hline$T I n_{i}$ & Um usuário adiciona uma bicicleta à estação $S_{i}$. \\
\hline$T W I n_{i}$ & $\begin{array}{l}\text { Um usuário que aguardava por vagas de bicicletas adi- } \\
\text { ciona uma bicicleta à estação } S_{i} \text {. }\end{array}$ \\
\hline$T A d d_{i}$ & O veículo adiciona uma bicicleta à estação $S_{i}$. \\
\hline TRem $_{i}$ & O veículo remove uma bicicleta à estação $S_{i}$. \\
\hline$T E_{j i}$ & $\begin{array}{l}\text { O veículo termina o balanceamento na estação } S_{i} \text { pela } \\
\text { ocorrência da condição } j \text {. }\end{array}$ \\
\hline $\operatorname{TInd}_{i j}$ & O usuário sai de $S_{i}$ com $S_{j}$ como destino. \\
\hline$T_{i j}$ & $\begin{array}{l}\text { O usuário que chega na estação } S_{i} \text { cheia escolhe } S_{j} \text { como } \\
\text { novo destino. }\end{array}$ \\
\hline$T t_{i j}$ & $\begin{array}{l}\text { Modela o tempo gasto pelo usuário para se deslocar da } \\
\text { estação } S_{i} \text { para a } S_{j} \text {. }\end{array}$ \\
\hline $\operatorname{TCarInd}_{i C}$ & O veículo sai de $S_{i}$ para o centro de redistribuição. \\
\hline TCarInd $_{i j}$ & O veículo se move da estação $S_{i}$ para $S_{j}$. \\
\hline TCarInd $d_{j}$ & O veículo sai do centro de redistribuição para $S_{j}$. \\
\hline TtCar $_{i C}$ & $\begin{array}{l}\text { Modela o tempo gasto pelo veículo para se deslocar da } \\
\text { estação } S_{i} \text { para o centro de redistribuição. }\end{array}$ \\
\hline$T t C a r_{i j}$ & $\begin{array}{l}\text { Modela o tempo gasto pelo veículo para se deslocar da } \\
\text { estação } S_{i} \text { para } S_{j} \text {. }\end{array}$ \\
\hline $\operatorname{TtCar}_{C j}$ & $\begin{array}{l}\text { Modela o tempo gasto pelo veículo para se deslocar do } \\
\text { centro de redistribuição } S_{j} \text { para a estação. }\end{array}$ \\
\hline
\end{tabular}

enquanto $m\left(P B S_{j}\right)=C_{j}$, conclui-se que a estação $S_{j}$ está cheia. Nessa situação, o usuário deverá escolher entre aguardar uma vaga na estação, ação representada pelo disparo da transição $T_{j j}$, ou escolher uma outra estação $S_{k}$ como destino, representada pelo disparo da transição $T_{j k}$. A escolha entre as transições $T_{j k}$ também é feita estocasticamente a partir dos valores de $\Lambda_{i}\left(T_{j k}, P I n_{j}\right)$. Caso $T_{j k}$ seja disparada, a ficha irá se mover para $P R_{j k}$, e caso $T_{j j}$ seja disparada, a ficha irá se mover para $P W i n_{j}$, lugar que representa que o usuário está aguardando uma vaga de bicicleta na estação. Note que, nesse caso, uma ficha será removida de $\mathrm{PInhib}_{j}$, impedindo que outros usuários depositem bicicletas na estação antes primeiro do usuário. Quando uma bicicleta for retirada da estação $S_{j}, P E S_{j}$ ganhará uma ficha, o que permitirá o disparo de $T W i n_{j}$, retirando uma ficha de $P W i n_{j}$ e de $P E S_{j}$ e adicionando uma ficha em $P B S_{j}$ e $P I n h i b_{j}$, simulando que o usuário devolveu a bicicleta. 
- Sub-rede do controle de estações (Lugares vermelhos). Essa sub-rede representa os componentes do modelo envolvidos na reposição/retirada de bicicletas em cada estação realizada pelo veículo de redistribuição. Assim como as estações, os lugares PBSCar e PESCar representam, respectivamente, a quantidade de bicicletas que ocupam o veículo de redistribuição e as vagas disponíveis no veículo. Para verificar em qual estação o veículo está efetuando o balanceamento, é utilizado o lugar $\mathrm{PCar}_{i}$, que indica que o veículo está realizando o balanceamento da estação $S_{i}$. Durante o balanceamento da estação $S_{i}$, o veículo é capaz de executar dois tipos de operações:

(i) Operação de adição de bicicletas na estação. Realizada pela transição $T A d d_{i}$, cuja habilitação ocorre quando a quantidade de bicicletas em $S_{i}$ for menor que o seu número de reordenação $R_{i}$ e se o veículo de redistribuição possuir pelo menos uma bicicleta.

(ii) Operação de remoção de bicicletas na estação: Realizada pela transição TRem $_{i}$, cuja habilitação ocorre quando a quantidade de bicicletas em $S_{i}$ for maior que o seu número de reordenação $R_{i}$ e se o veículo possuir pelo menos uma vaga de bicicleta disponível.

Essas operações são feitas com o objetivo de igualar a quantidades de bicicletas das estações com seus respectivos números de reordenação $R_{i}$. Porém, durante o balanceamento o veículo pode não ser capaz de balancear completamente uma estação. nesse caso, o modelo considera o balanceamento da estação $S_{i}$ pelo veículo de redistribuição como finalizado na ocorrência de uma das seguintes condições: (a) Quando o veículo de redistribuição estiver cheio e a quantidade de bicicletas na estação $S_{i}$ for maior que o número de reordenação $R_{i}$; (b) quando o veículo de redistribuição estiver vazio e a quantidade de bicicletas na estação $S_{i}$ for menor do que o número de reordenação $R_{i}$; (c) quando o veículo foi capaz de balancear completamente a estação, que ocorre quando o número de bicicletas na estação $P B S_{i}$ é igual ao número de reordenação $R_{i}$. Caso uma dessas condições ocorra, uma transição $T E_{j i}$ dispara, movendo a ficha do veículo de redistribuição para $P E_{i}$.

- Sub-rede do fluxo do veículo de redistribuição(Lugares verdes). Representa a movimentação do veículo entre as estações e o centro de redistribuição. No início, o veículo está estacionado no centro de redistribuição, o que é representado pela marcação $m\left(\operatorname{PCar}_{C}\right)=1$, cuja ficha é deslocada entre os lugares da sub-rede para representar o estado do veículo do balanceamento. Para decidir quando o veículo deve sair do centro e para qual estação ele deve ir para fazer seu primeiro balanceamento, é utilizada um controle externo para escolher, através dos $\operatorname{Esc}\left(\right.$ TCarInd $\left._{C i}, \operatorname{PCar}_{C}\right)$, qual transição TCarInd $_{C i}$ deve disparar, começando o movimento do veículo para a estação escolhida $S_{i}$. Decorrido um intervalo de tempo definido estocasticamente, a transição temporizada $\operatorname{TtCar}_{C i}$ dispara, transmitido a ficha para o lugar $\mathrm{PCar}_{i}$, representando que o veículo chegou a estação $S_{i}$. Quando o balanceamento da estação for finalizado, a sub-rede do controle de estações dispara a transição $T E_{k i}$, movendo a ficha para $P E_{i}$, representando que deve ser escolhido o próximo destino do veículo através de $\operatorname{Esc}\left(\operatorname{TCarInd}_{i j}, P E_{i}\right)$. Caso o destino escolhido seja outra estação $S_{j}$, a transição TCarInd $d_{i j}$ dispara, continuando o balanceamento, e caso o destino seja o centro de redistribuição, TCarInd ${ }_{i C}$ é escolhido para disparar e após a passagem de um tempo estocástico, a ficha que representa o veículo volta para $\mathrm{PCar}_{C}$.

\section{MATRIZES DE INCIDÊNCIA DO MODELO PROPOSTO PARA UM SPCB COM $N$ ESTAÇÕES}

Com a RPEGCE definida, é possível descrever sua dinâmica através da equação $m_{k+1}(\underline{p})=m_{k}(\underline{p})+A \sigma(\underline{t})$, em que $A=$ Post - Pre, e $p$ e $\underline{t}$ são, respectivamente, os vetores dos lugares e das transições da rede de Petri. Para tanto, os lugares são agrupados da seguinte forma:

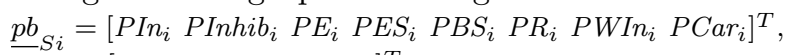

$\underline{\underline{p r}}_{S i}=\left[\begin{array}{llll}P R_{i 1} & P R_{i 2} & \ldots & P R_{i N}\end{array}\right]^{T}$,

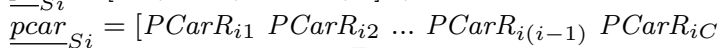

$\left.\overline{P C a r R}_{i(i+1)} \ldots P C a r R_{i N}\right]^{T}$,

$\underline{p}_{S i}=\left[\underline{p b}_{S i}^{T} \underline{p r}_{S i}^{T} \underline{p c a r}_{S i}^{T}\right]^{T}$ em que $i \in\{1,2, \ldots, N\}$,

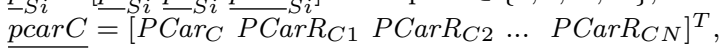
$\underline{\text { pcar }}=[\text { PBSCar PESCar }]^{T}$.

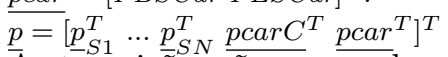

As transiçõ̃es são agrupadas nos vetores:

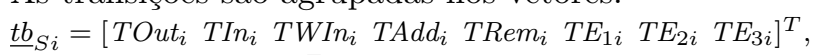

$\underline{\underline{t r}}_{S i}=\left[\begin{array}{llll}T_{i 1} & T_{i 2} & \ldots & T_{i N}\end{array}\right]^{T}$,

$\underline{\underline{t i}}_{S i}=\left[\begin{array}{llll}\operatorname{TInd}_{i 1} & \operatorname{TInd}_{i 2} & \ldots & \operatorname{TInd}_{i N}\end{array}\right]^{T}$,

$\underline{t t}_{S i}=\left[\begin{array}{llll}T t_{i 1} & T t_{i 2} & \ldots & T t_{i N}\end{array}\right]^{T}$,

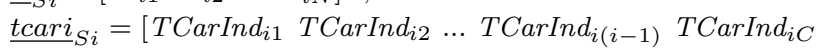

$\left.\operatorname{TCarInd}_{i(i+1)} \ldots \operatorname{TCarInd}_{i N}\right]^{T}$,

$\underline{t t c a r}_{S i}=\left[\begin{array}{lllll}\text {TtCar}_{i 1} & \operatorname{TtCar}_{i 2} & \ldots & \text { TtCar }_{i(i-1)} & \text { TtCar }_{i C}\end{array}\right.$

$\left.\operatorname{TtCar}_{i(i+1)} \cdots \operatorname{TtCar}_{i N}\right]^{T}$,

$\underline{t}_{S i}=\left[\underline{t b}_{S i}^{T} \underline{t r}_{S i}^{T} \underline{t i}_{S i}^{T} \underline{t t}_{S i}^{T} \underline{t c a r i}_{S i}^{T} \underline{t t c a r}_{S i}^{T}\right]^{T}$.

em que $i \in\{1,2, \ldots, N\}$,

$\underline{t \operatorname{tarC}}=\left[\begin{array}{lllll}T C a r_{C 1} & \operatorname{TCar}_{C 2} & \ldots & \operatorname{TCar}_{C N}\end{array}\right]^{T}$,

$\underline{t t c a r C}=\left[\begin{array}{lllll}T t C a r_{C 1} & T t C a r_{C 2} & \ldots & T_{t C a r_{C N}}\end{array}\right]^{T}$,

$\underline{t}=\left[\underline{t}_{S 1}^{T} \cdots \underline{t}_{S N}^{T}{\underline{t c a r} C^{T}}_{\underline{t t c a r} C^{T}}\right]$ Com os vetores definidos, é possível descrever as matrizes Pre e Post em função de blocos de matrizes:

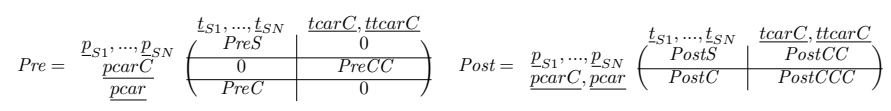

A seguir, são descritos cada um dos blocos e sub-blocos pertencentes às matrizes Pre e Pos.

- PreS: Matriz diagonal por blocos de dimensões $\left(2 N^{2}+\right.$ $8 N) \times\left(5 N^{2}+8 N\right)$ que pode ser escrita como PreS $=$ blockdiag $\left\{\right.$ Pre $_{1}$ Pre $_{2} \ldots$ Pre $\left.S_{N}\right\}$

- Pre $S_{i}$ : Matriz de dimensões $(2 N+8) \times(5 N+8)$ de uma estação $S_{i}$ definida como:

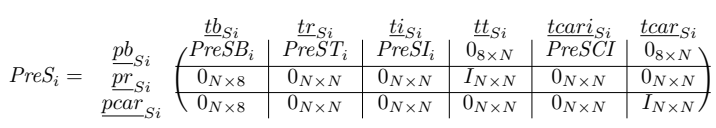

- $\operatorname{Pre} S B_{i}$ : Matriz de dimensões $8 \times 8$ de uma estação $S_{i}$ definida como:

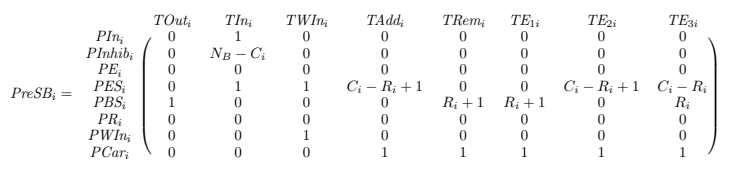

- $\operatorname{Pre} S T_{i}$ : Matriz de dimensões $8 \times N$ de uma estação $S_{i}$. Nela, todas as $N$ colunas $j$ são definidas como:

$\left[\begin{array}{llllllll}1 & 0 & 0 & 0 & C_{i} & 0 & 0 & 0\end{array}\right]^{T}$ se $i \neq j ;\left[\begin{array}{llllllll}1 & 1 & 0 & 0 & C_{i} & 0 & 0 & 0\end{array}\right]^{T}$ se $i=j$ 
- $\operatorname{Pre}_{S} I_{i}$ : Matriz de dimensões $8 \times N$ de uma estação $S_{i}$. Nela, todos os elementos são nulos, exceto a sexta linha, cujos elementos são uns.

- $\operatorname{Pre} S C I_{i}$ : Matriz de dimensões $8 \times N$ de uma estação $S_{i}$. Nela, todos os elementos são nulos, exceto a terceira linha, cujos elementos são uns.

- PreC: Matriz de dimensões $2 \times\left(5 N^{2}+8 N\right)$. Ela é dividida nos seguintes sub-blocos de colunas:

$$
\operatorname{Pre} C=\left[\begin{array}{cccc}
\underline{t}_{S 1} & \underline{t}_{S 2} & \ldots & \underline{t}_{S N} \\
\operatorname{PreC} S_{1} & \operatorname{PreC} S_{2} & \ldots & \operatorname{PreCS} S_{N}
\end{array}\right]
$$

- $\operatorname{Pre} C S_{i}$ : Matriz de dimensões $2 \times(5 N+8)$ de uma estação $S_{i}$. Ela é definida como:

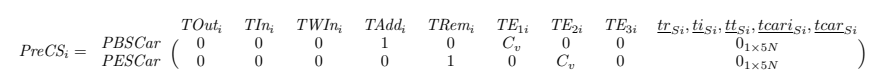

- Pre CC: Matriz de dimensões $(N+1) \times(2 N)$. A matriz é formada por dois sub-blocos de colunas:

$$
\operatorname{Pre} C C=\left[\begin{array}{ll}
\frac{\operatorname{tcar} C}{\operatorname{Pre} C C C} & \underline{\operatorname{ttcar} C} \\
\text { PreCCT}
\end{array}\right]
$$

- Pre $C C C$ : Matriz de dimensões $(N+1) \times N$. Nela, todos os elementos são nulos, exceto a primeira linha, cujos elementos são uns.

- PreCCT: Matriz de dimensões $(N+1) \times(N)$ de uma estação $S_{i}$. Ela é definida como:

$$
\operatorname{Pre} C C T=\left[0_{1 \times N} I_{N \times N}\right]^{T}
$$

- PostS: Matriz de dimensões $\left(2 N^{2}+8 N\right) \times\left(5 N^{2}+8 N\right)$ composta pela soma:

Post $S=$ blockdiag $\left\{\right.$ Post $_{1}$ Post $_{2} \ldots$ Post $\left._{N}\right\}+\left[\right.$ PostSc $_{1}$ PostSc $_{2} \ldots$ PostSc $\left.c_{N}\right]$

- Post $S_{i}$ : Matriz de dimensões $(2 N+8) \times(5 N+8)$ de uma estação $S_{i}$ definida como:

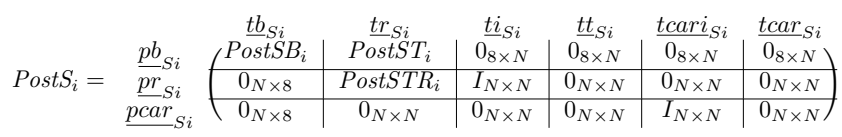

- PostSc $c_{i}$ : Matriz de dimensões $\left(2 N^{2}+8 N\right) \times(5 N+8)$ de uma estação $S_{i}$. É possível dividi-la nos sub-blocos de colunas:

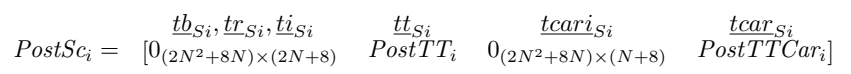

- PostSB $B_{i}$ : Matriz de dimensões $8 \times 8$ de uma estação $S_{i}$. Ela é definida como:

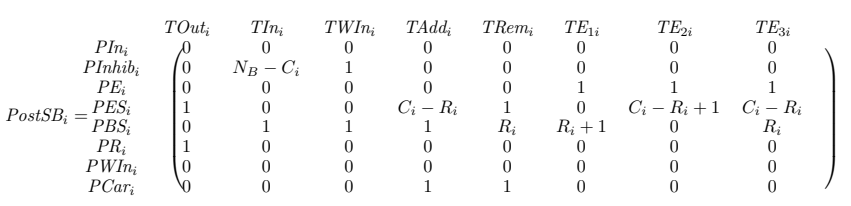

- PostST $T_{i}$ : Matriz de dimensões $8 \times N$ de uma estação $S_{i}$. Nela, todas as $\mathrm{N}$ colunas $j$ são definidas como:

$$
\left[\begin{array}{cccccccc}
0 & 0 & 0 & 0 & C_{i} & 0 & 0 & 0
\end{array}\right]^{T} \text { se } i \neq j ;\left[\begin{array}{llllllll}
0 & 0 & 0 & 0 & C_{i} & 0 & 1 & 0
\end{array}\right]^{T} \text { se } i=j
$$

- PostSTR $R_{i}$ : Matriz de dimensões $N \times N$ de uma estação $S_{i}$. Nela, todos os elementos da diagonal, exceto o elemento da $i$-ésima linha e $i$-ésima coluna, são unitários, os outros elementos da matriz são nulos.

- PostTT $T_{i}$ : Matriz de dimensões $\left(2 N^{2}+8 N\right) \times N$ de uma estação $S_{i}$. É possível dividi-la entre os sub-blocos:

$$
\operatorname{PostTT}_{i}=\left[\begin{array}{cccc}
\underline{p}_{S 1} & \underline{p}_{S 2} & \ldots & \underline{p}_{S N} \\
\text { PostTT }_{i 1} & \text { PostTT } & \ldots & \text { PostT }_{i N}
\end{array}\right]^{T}
$$

- PostTT $T_{i j}$ : Matriz de dimensões $(2 N+8) \times N$ das estações $S_{i}$ e $S_{j}$. Nela, todos elementos são nulos exceto o elemento da primeira linha e $j$-ésima coluna, cujo valor é um.

- PostTTCar : Matriz de dimensões $\left(2 N^{2}+8 N\right) \times N$ da estação $S_{i}$. É possível dividi-la entre os sub-blocos:

$$
\text { PostTTCar }_{i}=\left[\begin{array}{cccc}
\underline{p}_{S 1} & \underline{p}_{S 2} & \ldots & \underline{p}_{S N} \\
\text { PostTTCar }_{i 1} & \text { PostTTCar }_{i 2} & \ldots & \text { PostTTCar }_{i N}
\end{array}\right]^{T}
$$

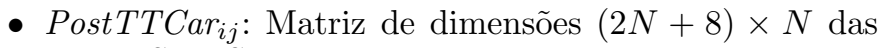
estações $S_{i}$ e $S_{j}$. Nela, se $i=j$, todos os elementos são nulos. Caso contrário, todos os elementos são nulos exceto o da oitava linha e $j$-ésima coluna, cujo valor é um.

- PostC: Matriz de dimensões $(N+3) \times\left(5 N^{2}+8 N\right)$. É possível dividi-la nos sub-blocos:

$$
\text { PostC }=\left[\begin{array}{cccc}
\underline{t}_{S 1} & \underline{t}_{S 2} & \ldots & \underline{t}_{S N} \\
\text { PostCS } & \text { PostCS } & \cdots & \text { PostCS }
\end{array}\right]
$$

- PostCS $S_{i}$ : Matriz de dimensões $(N+3) \times(5 N+8)$ de uma estação $S_{i}$. Ela é definida como:

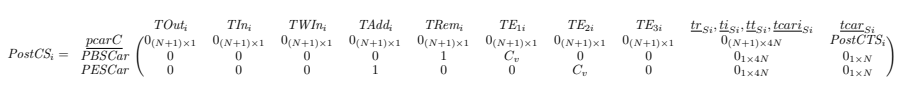

- PostCTS $S_{i}$ : Matriz de dimensões $(N+1) \times N$ da estação $S_{i}$. Nela, todos os elementos são nulos exceto o elemento da primeira linha e $i$-ésima coluna, cujo valor é um.

- PostCC: Matriz de dimensões $\left(2 N^{2}+8 N\right) \times 2 N$. É possível dividi-la entre os sub-blocos:

$$
\text { PostCC } \left.=\quad \begin{array}{cccc}
\underline{p}_{S 1} & \underline{p} S_{2} & \ldots & \underline{p} S N \\
\text { PostC } C_{1} & \text { PostC } C_{2} & \ldots & \text { Post } C C_{N}
\end{array}\right]^{T}
$$

- Post $C C_{i}$ : Matriz de dimensões $(8+2 N) \times 2 N$ da estação $S_{i}$. Nela, todos os elementos são nulos, exceto o elemento da oitava linha e da coluna $(N+i)$, cujo valor é um.

- PostCCC: Matriz de dimensões $(N+3) \times 2 N$. Ela é definida como:

$$
\left.\begin{array}{ccc}
\operatorname{PCar}_{C} & \underline{t \text { tarC }} & \frac{t t c a r C}{0_{1 \times N}} \\
0_{1 \times N} \\
I_{N \times N} & 0_{N \times N} \\
0_{2 \times N} & 0_{2 \times N}
\end{array}\right)
$$

Com as matrizes Pre e Pre definidas, é possível calcular que o número de lugares é $n_{P}=2 N^{2}+9 N+3$ e o número de transições é $n_{T}=5 N^{2}+10 N$.

\section{ALGORITMOS DE BALANCEAMENTO DE SPCB}

Como o modelo proposto neste trabalho permite a implementação de diferentes estratégias, neste trabalho serão apresentadas as três estratégias de balanceamento.

(i) Estratégia simples que realiza um balanceamento cíclico: A estratégia consiste em traçar uma rota 
constante em que o veículo visita cada estação sequencialmente durante suas rondas feitas em intervalos fixos de tempo.

Nessa estratégia, quando o veículo está no centro de redistribuição, em um certo momento o agente externo decide que ele deve iniciar a ronda e e se deslocar para a primeira estação. Após o veículo terminar a redistribuição em uma estação $S_{i}$, o agente externo escolhe a estação $S_{i+1}$ como próximo destino. Finalmente, na última estação, para finalizar a redistribuição, o agente externo define que o veículo deve retornar para o centro de redistribuição.

(ii) Estratégia adaptativa baseado no algoritmo de busca por força bruta: Com o intuito de evitar que o veículo não visite estações que não precisem ser balanceadas ou que não podem trocar bicicletas com o veículo a estratégia, no momento da decisão da próxima estação que o veículo deve visitar, é avaliado qual escolha é mais benéfica para o sistema utilizado o indicador $D B$, que representa o total de movimentos de bicicletas que devem ser realizados para estabilizar completamente a quantidade de bicicletas em cada estação com seus respectivos números de reordenação. O indicador $D B$ é descrito como: $D B=\sum_{i=1}^{N}\left|m\left(P B S_{i}\right)-R_{i}\right|$. Portanto, $D B$ indica o quão instável o sistema está.

Para construir uma rota para diminuir essa instabilidade, o algoritmo faz uma busca para verificar as próximas duas estações que o veículo deve visitar para estabilizar o máximo possível o sistema. Ele faz isso simulando, dentro do algoritmo externo, o que aconteceria com o número de bicicletas das estações se o veículo visitasse todas as possíveis duplas de estações de destino possíveis. Com cada possibilidade simulada, é calculado o valor de $D B$, para, com esses resultados, escolher o trajeto que causou o menor valor de $D B$ para ser o novo destino do veículo.

Neste trabalho, é considerado que o veículo realiza o mesmo número de viagens que o número de estações $N$ em cada ronda. Assim, em uma ronda, seriam analisadas $P T(N)=(4 N-3)(N-1)$ possibilidades. Note que, em cada busca realizada pelo algoritmo, é considerado somente as duas próximas estações, portanto, o número de possibilidades de cada busca é polinomial e igual a $2(N-1)$.

(iii) Estratégia preditiva que varia os números de reordenação de acordo com a demanda: A estratégia, diferente das outras, usa informações de possíveis estados futuros do sistema para alterar diretamente a ação de balanceamento de cada estação, variando o número de reordenação $R_{i}$ de cada estação de acordo com a demanda.

Em um sistema em que a demanda por bicicletas durante o dia é constante, poderia-se supor que o número ideal de bicicletas em cada estação seria metade da capacidade total dela, ou seja, para cada estação $S_{i}, R_{i}=C_{i} / 2$.

Alguns SPCBs, porém possuem horários específicos do dia em que ocorrem picos de utilização em estações específicas, causando uma alta demanda por bicicletas nelas e a necessidade de vagas disponíveis para as estações de destino dessas estações. Pode-se concluir que o ideal para essa situação é que as quantidades de bicicletas nas estações mencionadas sejam grandes enquanto as quantidades de bicicletas das estações de destino diminuam.

A partir dessa ideia, o algoritmo inicialmente atribui para cada $R_{i}$ metade do valor de $C_{i}$. Porém, no momento de pico de uma estação $S_{j}$, esse $R_{j}$ aumenta para $90 \%$ de $C_{j}$.

Além de aumentar o número de reordenação da estação $S_{j}$ com o pico, deve-se reduzir os números de reordenação das suas estações de destino. Essa redução é feita utilizados os dados descritos nas funções $\Lambda_{i}\left(\operatorname{TInd}_{j k}, P R_{j}\right)$. Assim, para cada estação $S_{k}$ que não possua um pico no momento, seu novo número de reordenação $R_{k}^{\prime}$ é reduzido de acordo com a equação $R_{k}^{\prime}=R_{k}-40 \% C_{j} \Lambda_{i}\left(\operatorname{TInd}_{i j}, P R_{i}\right)$.

Como as rondas de redistribuição começam em intervalos fixos de tempo, é recomendável realizar pelo menos uma ronda com os números de reordenação modificados antes do horário de pico. Por isso, os números de reordenação são modificados de um momento antes da ocorrência do pico com o mesmo intervalo de tempo que as rondas até o horário em que o pico acontece.

\section{IMPLEMENTAÇÃO DO ALGORITMO DE SIMULAÇÃO DO MODELO}

Com o modelo do SPCB, é possível implementar um algoritmo capaz de simular o funcionamento de do SPCB e avaliá-lo. Neste artigo, será implementado o modelo e o simulador do SPCB do Integra UFRJ, localizado na Ilha do Fundão da cidade do Rio de Janeiro.

Inicialmente, através dos blocos de matrizes descritos na seção 4, o simulador compõe as matrizes Pre e Post. Em seguida, é feito um vetor $\underline{m}_{S P C B}$, com $n_{P}$ elementos que representam a marcação da rede.

Quando uma das estratégias de balanceamento apresentadas altera os números de reordenação, as matrizes Pre e Post são recalculadas com os novos números.

Apesar da maioria das marcações iniciais dos lugares serem nulas, algumas começam com valores diferentes de zero. Primeiro, são adicionadas as fichas que representam as quantidades de bicicletas em cada estação. Para isso, supondo que tenha sido escolhido o valor inicial de $N b I n i_{i}$ bicicletas para cada estação $S_{i}$, são adicionadas $N b \operatorname{Ini}_{i}$ fichas à marcação $m\left(P B S_{i}\right)$ e $C_{i}-N b \operatorname{Ini} i_{i}$ à $m\left(P E S_{i}\right)$. Além disso, devem ser adicionadas $N_{B}-C_{i}$ fichas ao lugar $P I n h i b_{i}$ de cada estação $S_{i}$. O veículo também possui os lugares $P B S C$ ar e PESCar que representam as quantidades de bicicletas no veículo que devem ser preenchidas de acordo com a quantidade inicial de bicicletas no veículo. O lugar $\mathrm{PCar}_{C}$ deve possuir uma ficha para indicar que o veículo se encontra em sua posição inicial.

Durante a simulação, é verificado quais transições devem disparar em cada passo $k$ da simulação. Nesse passo, é definido um vetor $\sigma_{k}$, de comprimento $n_{T}$, cujos elementos representam as transições do sistema e são ordenados de acordo com $\underline{t}$. Caso o elemento em uma posição $j$ do vetor seja um, significa que a transição desse elemento deve disparar uma vez. Definir completamente $\sigma_{k}$, porém, não é trivial, transições especiais, cujos disparos não ocorrem apenas com suas habilitações. Assim, as transições especiais são divididas entre os grupos: 
(i) Transições temporizadas estocásticas: Como todas as transições temporizadas da rede são estocásticas, é atribuída, para cada transição temporizada $t_{j}$, uma função $f d p_{t_{j}}$ em $\Lambda_{d}\left(t_{j}\right)$ para sortear os tempos, em segundos, que levam para as transições dispararem após se tornarem habilitadas. Na rede proposta por este trabalho, todas as funções das transições temporizadas são distribuições uniformes, ou seja, para definir o tempo de disparo da transição, é escolhido um valor aleatório entre um tempo mínimo e máximo de forma uniforme.

A partir das funções $\Lambda_{d}($.$) definidas, torna-se possível criar$ uma lista que, dada a habilitação de uma transição temporizada, adiciona à si um elemento contendo o identificador da transição e o tempo que foi sorteado através da função. A cada passo do simulador, o valor de todos os tempos dos elementos da lista são reduzidos por um segundo. Conforme os elementos dessa lista se tornam menores ou iguais a zero, as transições relativas aos elementos disparam e o elemento é removido da lista.

(ii) Transições imediatas que estão envolvidas em conflitos solucionados estocasticamente: Conflitos entre transições imediatas podem ser solucionados através de um sorteio realizado utilizando probabilidades definidas para cada transição através de $\Lambda_{i}($.$) . No modelo, os dois$ grupos de conflitos são resolvidos dessa forma: o conflito entre as transições $\operatorname{TInd}_{i j}$ e o conflito entre $T_{i j}$.

Para realizar o sorteio de um desses conflitos entre um grupo de transições, primeiro é feita uma soma cumulativa dos valores $\Lambda_{i}($.$) das transições do conflito, de tal forma$ que resulte em um vetor ordenado. Após isso, é sorteado um número aleatório de 0 a 1 , cujo valor indica que a posição do primeiro elemento que é maior do que o número aleatório representa qual das transições deve disparar.

(iii) Transições imediatas que estão envolvidas em conflitos solucionados por meio de um controle externo: Alguns dos conflitos entre transições imediatas da rede podem ser resolvidos através de um agente externo. Neste trabalho existem dois tipos de agentes externos: o conflito degenerado da transição TOut $_{i}$ e as transições TCarInd $_{i j}$, tal que $i, j \in\{1,2, \ldots, N, C\}$, em que $C$ é o centro de redistribuição e $i \neq j$, que são resolvidos de acordo com os algoritmos apresentados na seção 5 .

Durante a inicialização do primeiro agente externo, que avalia o disparo das transições TOut $_{i}$ de cada estação $S_{i}$, é definida a matriz $\operatorname{Tim}_{u}$, de dimensões $N \times T s$, que representa a chegada de um usuário na estação da linha $i$ no tempo, em segundos, da coluna $j$, em que Ts é a duração de tempo total da simulação. Para realizar isso, deve-se dividir o número de pessoas que usam o sistema em números de pessoas que retiram bicicletas das estações durante os horários de pico, representado pelo vetor $N_{p}$, e números de pessoas que alugam bicicletas normalmente ao longo do dia, representado por $N_{u}$, cujos elementos representam cada estação.

Para alocar os usuários de $S_{i}$ representados em $N_{u}$, é feito, para cada usuário, um sorteio uniforme de um número $j$ aleatório de 1 até $T s$. O número sorteado representa a linha $i$ e coluna $j$ do elemento da matriz Timm $_{u}$ cujo valor é alterado para um.
O mesmo tipo de sorteio ocorre para os usuários de $N_{p}$, porém, ele é realizado com a distribuição de Poisson, em que a probabilidade de cada número $k$, entre o intervalo de 1 a Ts, ser escolhido é, em função da constante $\alpha$, que representa os horários de pico: $\frac{\exp ^{-\alpha} \alpha^{k}}{k !}$. Se uma estação possuir mais de um pico, $N_{p}(i)$ é dividido entre os picos.

Com os horários de entrada no sistema dos usuários definidos, é verificado, em cada passo $k$ da simulação e para cada estação $S_{i}$, se $\operatorname{Tim}_{u}(i, k)$ é igual a um. Caso a verificação seja positiva, o simulador tenta disparar TOut . Porém, Caso a transição não esteja habilitada, esse usuário é movido para uma fila, com um tamanho fixo e determinado, onde ele aguarda até que TOut $_{i}$ esteja habilitada para disparar. Se um usuário chegar na estação enquanto sua fila está cheia, é considerado que um dos usuários desistiu de esperar por uma bicicleta.

\section{PARÂMETROS DE ENTRADA DO SIMULADOR}

O simulador utiliza diferentes parâmetros de entrada para que seja possível implementar diferentes SPCBs. Para apresentar essas variáveis, elas descritas a seguir:

- Ts: Duração de tempo total da simulação, em segundos.

- init $_{\mathbf{T}}$ : Tempo inicial do sistema, em horas.

- N: Número total de estações do SPCB a ser simulado.

- PCheg: Matriz de dimensões $N \times\left(P_{\text {Max }}+1\right)$ que contém os horários, em segundos, de todos os picos presentes nas estações, representadas pelas linhas da matriz.

- $\mathbf{N}_{\mathbf{u}}$ e $\mathbf{N}_{\mathbf{p}}$ : Vetores de dimensão $N$ cujos elementos da posição $i$ representam, respectivamente, a quantidade de usuários que alugam bicicletas ao longo do dia e usuários que alugam bicicletas durante os picos da estação $S_{i}$.

- DIda: Matriz de dimensões $N \times N$ em que cada elemento representa a chance do usuário que sai da estação da linha $i$ escolher como destino a estação da coluna $j$.

- DReIda: Matriz de dimensões $N \times N$ em que cada elemento representa a chance do usuário, após encontrar a estação da linha $i$ cheia, escolher como destino a estação da coluna $j$.

- C: Vetor de tamanho $N$ que contém a capacidade de armazenamento de bicicletas em cada estação $S_{i}$.

- $\mathbf{C}_{\mathbf{v}}$ : Número que representa a capacidade de armazenamento de bicicletas do veículo de redistribuição.

- NbIni: Vetor de tamanho $N$ que contém a quantidade inicial de bicicletas em cada estação $S_{i}$.

- $\mathbf{N b I n i}_{\mathbf{v}}$ : Variável que representa a quantidade inicial de bicicletas no veículo de redistribuição.

- $\mathbf{T t M i n}$ b $\mathbf{T} \mathbf{t} \mathbf{M a x}_{\mathbf{b}}$ : Matrizes $N \times N$ que representam, respectivamente, os menores e os maiores tempos que levam para o usuário se locomover da estação da linha $i$ até a estação da coluna $j$.

- $\mathbf{T t M i n}$ e TtMax $_{\mathbf{c}}$ : Matrizes $N \times N$ que representam, respectivamente, os menores e os maiores tempos que levam para o veículo se mover da linha $i$ para a coluna $j$, em que $i, j \in\left\{C, S_{1}, S_{2}, \ldots, S_{N}\right\}$ e $i \neq j$.

- Ron $_{\text {St: }}$ Variável que representa o tempo, em segundos, que a primeira ronda do veículo de redistribuição é realizada durante o dia.

- Ron Car: Variável que representa o intervalo que o veículo aguarda entre as rondas.

- Max Fila: Vetor de tamanho $N$ que representa o número máximo de pessoas que aguaram em cada estação $S_{i}$ por bicicletas, caso ela esteja vazia. 
Tabela 4. Indicadores médios das 50 simulações do sistema sem balanceamento.

\begin{tabular}{|c|c|c|c|c|c|c|c|c|c|}
\hline & casos & $S_{1}$ & $S_{2}$ & $S_{3}$ & $S_{4}$ & $S_{5}$ & $S_{6}$ & $S_{7}$ & $S_{8}$ \\
\hline Porcentagem & (i) & 13,5 & 2,5 & 20,4 & 24,7 & 10,9 & 19,2 & 12,9 & 3,9 \\
\hline de tempo & (ii) & 7,3 & 0,1 & 1,8 & 9,1 & 1,0 & 15,2 & 2,5 & 1,6 \\
\hline vazias & (iii) & 4,9 & 0,1 & 2,2 & 4,2 & 0,9 & 1,0 & 0,6 & 1,0 \\
\hline Porcentagem & (i) & 19,3 & 7,4 & 14,5 & 5,7 & 14,2 & 7,8 & 0,9 & 19,4 \\
\hline de tempo & (ii) & 2,5 & 0,4 & 6,3 & 2,2 & 1,8 & 2,6 & 0,3 & 1,1 \\
\hline cheias & (iii) & 0,3 & 0,1 & 6,9 & 5,3 & 2,1 & 6,3 & 0,0 & 1,3 \\
\hline Porcentagem & (i) & \multicolumn{8}{|c|}{11,0} \\
\hline de usuários & (ii) & \multicolumn{8}{|c|}{9,7} \\
\hline perdidos & (iii) & \multicolumn{8}{|c|}{2,3} \\
\hline
\end{tabular}

\section{INDICADORES PARA A AVALIAÇÃO DOS DESEMPENHOS DOS SISTEMAS SIMULADOS}

Para avaliar como a eficiência do SPCB varia, torna-se necessário estabelecer os indicadores:

- $\mathbf{t E}_{\mathbf{i}}$ : Indica a porcentagem de tempo que cada estação $S_{i}$ permaneceu vazia durante o funcionamento do sistema.

- $\mathbf{t F}_{\mathbf{i}}$ : Indica a porcentagem de tempo que cada estação $S_{i}$ permaneceu cheia durante o funcionamento do sistema.

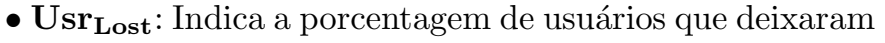
de usar o sistema por causa de estações vazias, ao encontrarem grandes filas esperando por bicicletas nas estações.

\section{RESULTADOS}

Nesta seção, a partir dos parâmetros do sistema do Integra UFRJ, são realizadas diversas simulações de três tipos de combinações das estratégias de balanceamento: (i) nenhuma estratégia de balanceamento; (ii) balanceamento cíclico com números de reordenação fixos; (iii) balanceamento adaptativo com números de reordenação adaptativo. A análise dos indicadores de uma simulação individual para cada estratégia não é confiável, por isso, cada estratégia foi simulada 50 vezes, resultando em 50 grupos de indicadores que resultam, através de uma média, em indicadores mais confiáveis do que indicadores obtidos por apenas uma simulação. Após todas as simulações, foram obtidos os indicadores médios da tabela 4 e o gráfico 2 das 3 primeiras simulações de cada estratégia contendo as quantidades de bicicletas nas estações e no veículo e a rota que o veículo fez.

\section{CONCLUSÃO}

Neste trabalho, foi proposto o modelo de RPEGCE, para descrever o funcionamento de um SPCB com um veículo. Além do modelo permitir a análise do desempenho de diferentes sistemas, ele torna possível a implementação de diferentes tipos de estratégias de balanceamento, cujo controle externo não precisa ser inserido na rede de Petri. Além disso, por estar implementado separadamente do simulador, o controle externo pode ser utilizado diretamente em sistemas reais.

Dentre as estratégias de balanceamento propostas no artigo, foi mostrado, por meio de simulação, que a estratégia (iii) foi a mais eficiente. A combinação da estratégia adaptativa com a estratégia preditiva otimizou simultaneamente o planejamento da ronda do veículo e a resposta do sistema aos picos de utilização. É importante ressaltar que o sucesso da estratégia ( iii) ocorreu quando todos os horários de pico são conhecidos; caso algum horário não seja conhecido, a eficiência da estratégia (iii) seria equivalente à obtida com a estratégia $(i i)$.
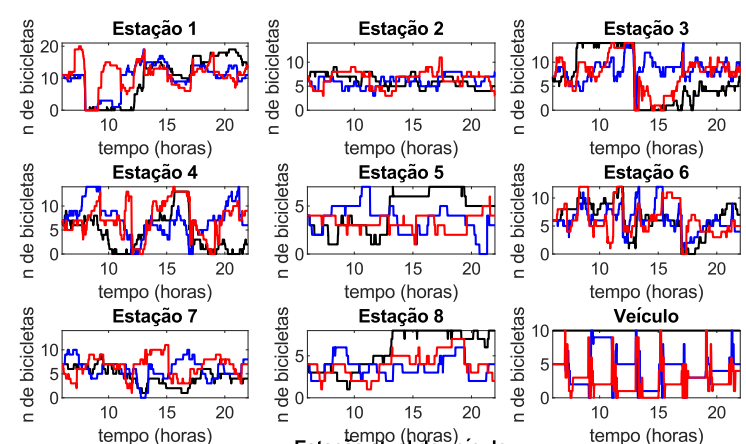

Estação 6
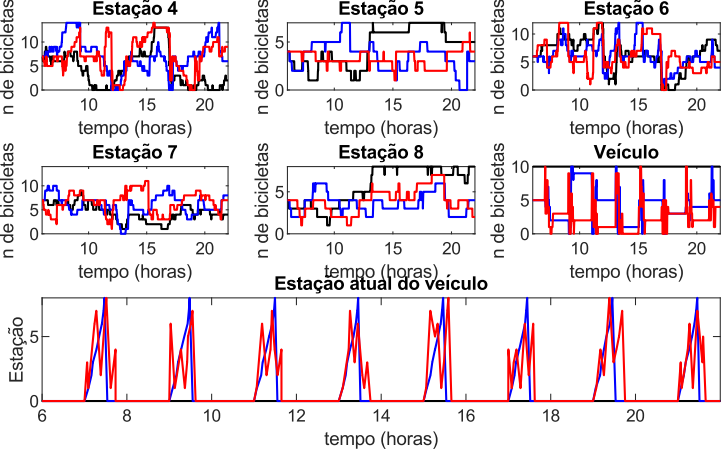

Figura 2. Quantidades de bicicletas nas estações e no veículo e a rota do veículo para a estratégia $i$ (preto), ii(azul) e $i i i$ (vermelho)

\section{REFERÊNCIAS}

Antunes, I., Carvalho, L.K., and Basilio, J.C. (2016). A stochastic petri net model for simulation-based performance analysis of public bicycle sharing systems. In 2016 IEEE International Conference on Automation Science and Engineering (CASE), 433-439.

Benarbia, T., Labadi, K., Omari, A., and Barbot, J. (2013). Balancing dynamic bike-sharing systems: A petri nets with variable arc weights based approach. In Control, Decision and Information Technologies (CoDIT), 112-117.

Chemla, D., Meunier, F., and Wolfler Calvo, R. (2013). Bike sharing system: solving the static rebalancing problem. Discrete Optimization, 10(2), 120-146.

David, R. and Alla, H. (1994). Petri nets for modeling of dynamic systems: A survey. Automatica, 30(2), 175-202.

Labadi, K., Benarbia, T., Barbot, J., Hamici, S., and Omari, A. (2014). Stochastic petri net modeling, simulation and analysis of public bicycle sharing systems. IEEE Transactions on Automaton Science and Engineering, $\mathrm{PP}, 1-16$.

Labadi, K., Darcherif, A.M., Hamaci, S., and Benarbia, T. (2012). Petri Nets Models for Analysis and Control of Public Bicycle-Sharing Systems. INTECH Open Access Publisher.

Murata, T. (1989). Petri nets: Properties, analysis and applications. Proceedings of the IEEE, 77(4), 541-580.

Shu, J., Chou, M., Liu, Q., Teo, C.P., and Wang, I.L. (2010). Bicycle-sharing system: deployment, utilization and the value of re-distribution. National University of Singapore-NUS Business School, Singapore.

Vogel, P., Greisera, T., and Mattfelda, D.C. (2011). Understanding bike-sharing systems using data mining: Exploring activity patterns. Procedia - Social and Behavioral Sciences, 20, 514-523.

Vogel, P. and Mattfeld, D.C. (2010). Modeling of repositioning activities in bike-sharing systems. In 12th World Conference on Transport Research (WCTR), Lisbon, Portugal. 